\title{
Defining the effective deformation of the vertebral column
}

\author{
Abtin Daghighi ${ }^{1}$, Hans Tropp $^{2}$ \\ ${ }^{1}$ Department of Mathematics and Mathematical Statistics, Umeá University, 90187 \\ Umeȧ, Sweden, e-mail: abtin.daghighi@umu.se \\ ${ }^{2}$ Department of Biomedical and Clinical Sciences, Division of Surgery, Orthopaedics \\ and Oncology, Linköping University, 58183 Linköping, Sweden, \\ e-mail: hans.tropp@liu.se
}

\section{SUMMARY}

The Cobb angle is calculated in the coronal plane, irrespective of vertebral rotation, lordokyphosis and local wedge properties of individual vertebrae other than the end plates used for the measurement. Rigorous threedimensional generalizations of the Cobb angle are complicated for at least two reasons. Firstly, the vertebral column is segmented, not continuous, making the choice of rigorous model ambiguous. Secondly, there exists an inherent curvature (in terms of thoracic kyphosis and lumbar lordosis) that may be considered physiologically healthy or 'normal'. When attempting to find a three-dimensional deviation measure, such normal sagittal curvature must be compensated for.

In this paper we introduce a three-dimensional local deformation parameter (which we call the local effective deformation) motivated by both biomechanics and the basic theory of spatial curves, and simultaneously introduce a technical procedure to estimate the parameter from CT scans using MPR (multi-phase reconstruction) in PACS (IDS-7). A detailed description of the proposed modelling of vertebral column deformation is given, together with a stepwise procedure to estimate the three-dimensional deformation (in terms of local effective deformation). As a deformation measure it requires knowledge about the natural healthy kypholordosis. A method is described by which such knowledge may be incorporated in future work.

Key words: Scoliosis, Three-dimensional deformation, Cobb angle, Space curve

\section{Background}

Many factors are logically involved when describing the spatial deformation of the vertebral column in a scoliotic curve (see Dansereau and Stokes (1988) 
and Stokes et al. (2009)). The Cobb angle is calculated in the coronal plane, irrespective of vertebral rotation, kypholordosis and local wedge properties of individual vertebrae other than the end-plates, whereas some classification systems, like the Lenke classification (2001), utilize an additional crude sagittal modifier. For background on the most common classification systems (Lenke et al. 2001) in adolescent idiopathic scoliosis, see e.g. Ovadia (2013). Some recent works on 3-D classification of spine deformation include Somoskeyöy et al. (2012), Sangole et al. (2009), Stokes (2009) and Kawakami et al. (2009). For a recent review on 3-D scoliosis classifications and on different existing methods for evaluation of spinal curvature, see e.g. Donzelli et al. (2015), Vrtorvec et al. (2009). We also mention the book edited by D'Amico et al. (1995) and Drerup and Hierholzer (1992) regarding shape estimation from frontal radiographs, where harmonic estimation is used. We believe also that the Lenke system is a good system in practice, but does not reflect the three-dimensional deformity near the apex in an optimal fashion (from a theoretical point of view, despite its sagittal modifier). Stokes (1994) presented a report on the terminology used in describing three-dimensional spinal deformity, where the notion of a 'vertebral body line' is used, and means a 'curved line' passing through the 'centroids' of each vertebral body. The work is appealing from a technical viewpoint as it promotes the application of the theory of space curves to scoliosis. The notion of curvature and torsion would obviously be rather useful in describing the three-dimensional deformation of a vertebral column, and if there were to exist a well-defined space curve associated to a given vertebra, then the local curvature and torsions of that curve could be investigated in a segment and render an appropriate measure of deformation for the corresponding segment of the vertebral column. The authors believe that there are ways to complement the notion of 'vertebral body line' or to introduce a similar notion that is more rigorous from a technical point of view. We believe that associating a space curve to a vertebral column from a discrete set of points is problematic from the perspective of uniqueness. For this reason we propose merely to postulate the existence of a unique most appropriate underlying curve, and then develop an algorithm that strives to approximate curvatures and torsions based upon appropriate anatomical reasoning. 


\section{Aim}

The aim of this paper is to introduce a local deformation parameter (which we call the local effective deformation) motivated by both biomechanics and the basic theory of spatial curves, and simultaneously introduce a technical procedure to estimate the parameter from CT scans. Apical rotation, wedge properties and tilt will all automatically be part of the manifested deformation measure. In such a model it will be clear that apical rotation, for example, is simply a part of the torsion of a postulated underlying space curve associated to the vertebral column. Obviously, a certain degree of kypholordosis is naturally present in normal anatomy. To account for this, one needs more detailed knowledge on what can be considered 'natural' local sagittal curvature, denoted $\hat{\kappa}$. In Section 7 we shall propose a method to estimate such natural curvatures, making it possible to use our parameter as a measure of deviation relative to a normal (or healthy) vertebral column. It is our intention to pursue such a method in future work (for comparison, we shall have to estimate a curvature, denoted Cobb associated deformation, derived from the Cobb angle and the end-plates used in the calculation of the Cobb angle; see Section 5).

\section{Method}

Our method for defining a three-dimensional definition of the deformation of the vertebral column is based upon a small set of base assumptions, and the definition obtained is manifested through analogy with the theory of space-curves. The technical procedure to estimate the descriptor from CT scans is based on the use of MPR (multi-phase reconstruction) in PACS (IDS-7). A detailed description of the proposed modelling of vertebral column deformation is given together with a stepwise procedure to estimate the three-dimensional deformation from CT scans. We call our deformation parameter the effective deformation. The idea is inspired by the use of a space curve associated to a given set of vertebra; then discrete analogues of the local curvature and torsions of that curve can be investigated in a segment and render an appropriate measure of deformation for the corresponding segment of the vertebral column. However, we believe that associating a space curve to a vertebral column from a discrete set of points is problematic from the perspective of uniqueness. For this reason we propose merely to postulate the existence of a unique most appropriate underlying curve, 
and then develop an algorithm that strives to approximate curvatures and torsions based upon appropriate anatomical reasoning. An exception clause is used in the case of anatomical anomalies which hinder the steps of the main algorithm, so that in such cases a method of modelling by artificial geometric shapes, detailed previously in Daghighi et al. (2018), is used. We shall describe how the main procedures are obtainable using modern roentgenological software. For our model we use the following postulates.

(P1) The axial vertebral rotation in a model healthy vertebral column is assumed to be zero for each vertebra. The procedure of calculating the Cobb angle applied to any level of a healthy vertebral column where the calculation is possible will render the angle zero.

(P2) There exists a unique $C^{\infty}$-smooth space curve associated to the segment L5-T1 of any given complete vertebral column, whose curvature and torsion are most appropriate for describing the three-dimensional deformation of the vertebral column segment.

(P3) A model healthy vertebral column has a natural sagittal kypholordosis; in particular we postulate that at each vertebral level there exists a number that describes the 'mean' of the random variable describing local curvature at that level, with respect to healthy vertebral columns.

\section{Results}

The result is a rigorous procedure for calculating a deformation parameter (local and global respectively) which is mathematically well-motivated from the theory of space curves. To present this resultant procedure we must thus give a detailed presentation and motivation of the steps involved.

\section{Proposed deformation parameter.}

The procedure we propose can be described by the following steps.

(1) To each vertebra from L5 to T1 we associate three points and three 2-dimensional planes. (The exclusion of $\mathrm{C} 1-\mathrm{C} 7$ is due to the use of certain anatomical landmarks in our procedure, the fact that often the cervical vertebrae are not included in the preoperative $\mathrm{CT}$, and our belief that this restriction is affordable due to the fact that usually the apex of a deformation does not lie close to the cervical vertebrae). 
(2) Using the three planes we obtain uniquely defined pairs of unit vectors (they will be used as 'tangent' and 'normal' vectors) at each of the three points.

(3) We work under the hypothesis (P2) that there exists a unique $C^{\infty}$ smooth curve, denoted $\gamma$, which passes through all position data points and at those points has the tangents and normals from previous steps, and in some sense best approximates the spatial deformation of the spine. Obviously, attempting actually to interpolate a large number of points will usually give unrealistic oscillations. For this reason we shall use discrete approximation. (Obviously it would be sufficient to require $C^{3}$-smoothness, but even in the case of $C^{\infty}$-smooth curves the problem of unique assignment is not straightforward due to abundance. On the other hand, real analyticity is too strong if there exist certain known curve segments that one believes should be part of the chosen representative.)

(4) To each of the points $p_{1}, p_{2}, p_{3}$ associated to a vertebral body we associate an estimate of the local curvature and local torsion of the hypothesized curve $\gamma$, given in vector form $\left(\kappa_{j}, \tau_{j}\right), j=1,2,3$, where $\kappa_{j}$ denotes the estimate of local curvature and $\tau_{j}$ the estimate of local torsion at $p_{j}$. This can be motivated based upon $(\mathbf{P 1})$. We will be able to do this for all except the end points corresponding to the bottom of $L 5$ and the top of $T 1$. According to Postulate (P3) there exist physiologically healthy curvatures $\hat{\kappa}_{j}$ at each $p_{j}, j=1,2,3$, and we replace $\kappa_{j}$ with $\operatorname{sign}\left(\kappa_{j}\right)\left|\kappa_{j}-\hat{\kappa}_{j}\right|$. We call the latter the local effective curvature.

Our deformation parameter with respect to each point is the absolute value $\left|\left(\kappa_{j}, \tau_{j}\right)\right|$ for all except the points associated with the bottom of L5 and top of T1.

(Exception clause) In the case of abnormal anatomy (e.g. congenital or previous operation with pedicle screws) we include a case exception, where the vertebra under consideration is modelled by the procedure described in detail in our previous work, Daghighi et al. (2018), Section 3.3, Figures 5 and 6.

Remark 4.1. The contribution of axial vertebral rotation, vertebral tilt and the wedge properties of each vertebra are incorporated in the choice of the planes associated to the vertebrae. Note also that in a healthy spine we 
postulate that the axial vertebral rotation and the local torsion of the curve equal zero, so that the contribution of $\tau$ in $|(\kappa, \tau)|^{2}=\kappa^{2}+\tau^{2}$ is assumed to be zero for healthy patients. However, we know that in healthy patients the local curvature is nonzero, due to the fact that there exists a natural kypholordosis. The possibility of estimating this in practice is discussed in Section 7.

Let us now give the details of each step.

\subsection{Step 1. (The points $p_{1}, p_{2}, p_{3}$ and associated planes $\Pi_{1}, \Pi_{2}, \Pi_{3}$ )}

Let $\eta_{1}^{\prime}$ and $\eta_{2}^{\prime}$ respectively denote points of the left and right superior vertebral notch (recall that the superior vertebral notch lies between the vertebral body and the superior articulate facet) such that the line defined by these points is tangential to the superior vertebral notch on both sides. Choose these points such that they divide the length of the superior margin of the notches approximately in half.

Let $\eta_{1}$ and $\eta_{2}$ denote the two points on the apical marginal ridge (top surface) of the vertebral body that have minimum Euclidean distance to $\eta_{1}^{\prime}$ and $\eta_{2}^{\prime}$ respectively.

Let $\eta_{3}^{\prime}$ and $\eta_{4}^{\prime}$ respectively denote the points of the left and right inferior vertebral notch of closest Euclidean distance to the most apical point $\eta_{0}$ of the vertebral groove, such that the line defined by $\eta_{3}^{\prime}, \eta_{4}^{\prime}$ is tangential to the inferior vertebral notch on both sides.

Let $\eta_{3}$ and $\eta_{4}$ denote the points on the caudal marginal ridge (bottom surface) of the vertebral body that have minimum distance to $\eta_{3}^{\prime}$ and $\eta_{4}^{\prime}$ respectively.

Let $q_{0}$ denote the mean of the two points $\frac{1}{2}\left(\eta_{1}+\eta_{3}\right)$ and $\frac{1}{2}\left(\eta_{2}+\eta_{4}\right)$, and let $p_{0}$ be the point on the vertebral groove of closest Euclidean distance to $q_{0}$. Let $\eta_{5}$ ( $\eta_{6}$ respectively) be the point on the line defined by $\left\{\eta_{1}, \eta_{3}\right\}$ $\left(\left\{\eta_{2}, \eta_{4}\right\}\right)$ of closest Euclidean distance to $p_{0}$. Denote by $\Pi_{1}$ the plane defined by $p_{0}, \eta_{5}, \eta_{6}$.

Figure 1 shows the choices of $p_{0}, \eta_{5}, \eta_{6}$ and thereby $\Pi_{1}$, for a lumbar vertebra. Isolate the slice of the vertebra in the plane $\Pi_{1}$. Postulating a theoretical slice symmetry of the vertebra, and attempting to find a practical analogue of a symmetry invariant line through $p_{0}$, we select (similarly as in the procedure described in Lam et al. (2008) and Ho et al. (1993)) the line, denoted $\ell$, in $\Pi_{1}$, obtained as follows: (i) Let $C_{r_{j}}$ be the circle in $\Pi_{1}$, centred at $p_{0}$ with radius $r_{j}>0, j=1, \ldots, 5$, where $r_{k}-r_{k+1}$ is a positive constant 

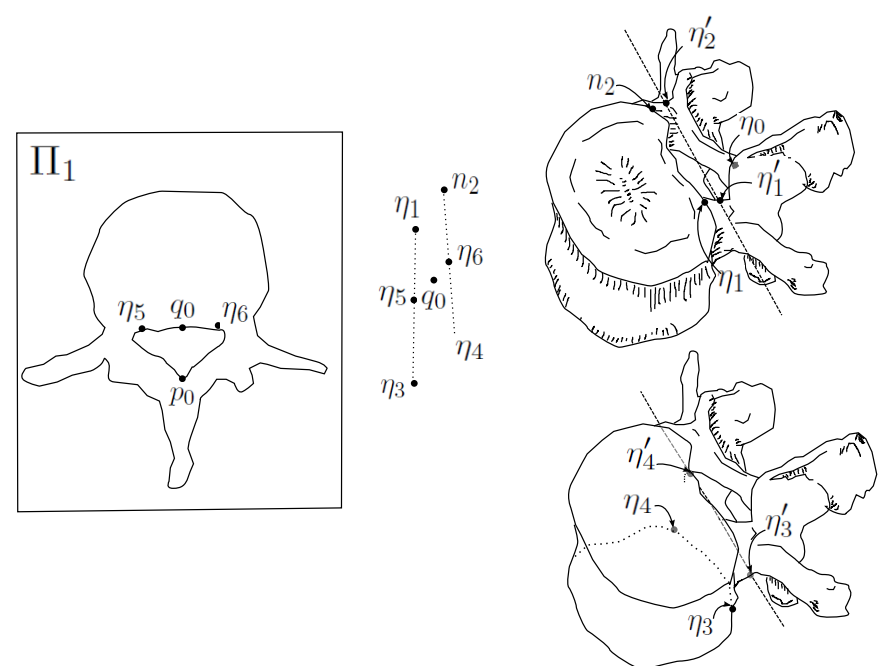

Figure 1. The choices of $p_{0}, \eta_{5}, \eta_{6}$ and thereby $\Pi_{1}$, for a lumbar vertebra. This is part of Step 1 (Section 4.1). Here we use the fact that any three non-collinear points define a unique two-dimensional plane (here $\Pi_{1}$ )

(independent of $k$ ) and $r_{1}$ is chosen such that the intersection of $C_{r_{1}}$ with the slice of the foramen vertebralis in $\Pi_{1}$ consists of precisely two points, $R_{1}^{1}, R_{2}^{1}$, on the posterior arc of the foramen. Since the $r_{j}$ are decreasing, we then obtain, for each $j$, that the intersection of $C_{r_{j}}$ with the slice of the foramen vertebralis in $\Pi_{1}$ consists of precisely two points, $R_{1}^{j}, R_{2}^{j}$, on the posterior arc of the foramen. (ii) For each $j=1, \ldots, 5$, let $\ell^{j}$ denote the bisector of the angle formed by the two vectors $\left(R_{1}^{j}-p_{0}\right),\left(R_{2}^{j}-p_{0}\right)$ originating at $p_{0}$. (iii) For each $j$ let $\Phi_{j}$ denote the midpoint of the intersection of $\ell^{j}$ with the slice of the vertebral body in $\Pi_{1}$, and let $\rho_{j}$ denote the radius of the largest disc that can be fitted into that slice with centre $\Phi_{j}$. Choose $\ell:=\ell^{J}$, where $\rho_{J}=\max _{j} \rho_{j}$. Choose $p_{1}$ as the midpoint of the intersection of $\ell$ with the slice of the vertebral body in $\Pi_{1}$.

Figure 2 shows the choices of $C_{r_{1}}, R_{1}^{1}, R_{2}^{1}, \Phi_{1}$ in the first iteration of the procedure for defining $p_{1}$.

Let $\Pi_{0}$ denote the plane passing through $p_{1}$ and spanned by $\left(p_{1}-p_{0}\right)$ and $\left(p_{1}-p_{0}\right) \times\left(\eta_{6}-\eta_{5}\right)$. Isolate the slice of the vertebra in the plane $\Pi_{0}$, and approximate the top (bottom) by a line segment denoted $\ell_{1}^{\prime}\left(\ell_{2}^{\prime}\right)$. Denote by $s_{1}\left(s_{2}\right)$ the midpoint of $\ell_{1}^{\prime}\left(\ell_{2}^{\prime}\right)$. Denote by $\hat{\Pi}_{j}$ the plane passing through $s_{j}$ and spanned by $\left(s_{j}-p_{1}\right)$ and $\left(s_{j}-p_{1}\right) \times\left(p_{1}-p_{0}\right), j=1,2$. Isolate the slice of the vertebra in the plane $\hat{\Pi}_{2}\left(\hat{\Pi}_{3}\right)$ and approximate the top (bottom) 


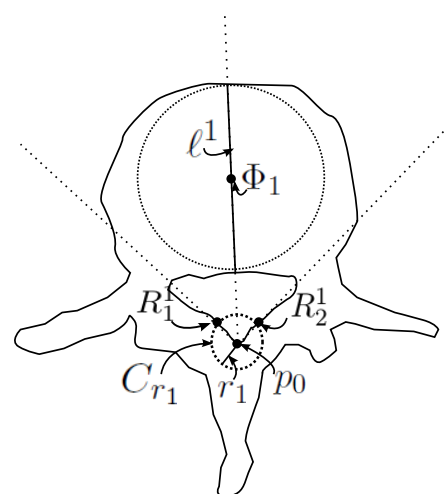

Figure 2. The choices of $C_{r_{1}}, R_{1}^{1}, R_{2}^{1}, \Phi_{1}$ in the first iteration of the procedure for defining $p_{1}$. This is part of Step 1 (Section 4.1). This process takes place in the plane $\Pi_{1}$ (see Figure 1) previously defined in Step 1. The notations are described in detail in the main text of Step 1

by a line segment denoted $\ell_{1}\left(\ell_{2}\right)$. The point $p_{2}$ is defined as the midpoint of $\ell_{1}$, and the point $p_{3}$ is defined as the midpoint of $\ell_{2}$. Denote by $v_{1}^{\prime}$, the unit direction vector of $\ell$. Denote by $v_{2}\left(v_{2}^{\prime}\right)$ the (up to sign) unit direction vector of $\ell_{1}\left(\ell_{1}^{\prime}\right)$. Denote by $v_{3}\left(v_{3}^{\prime}\right)$ the (up to sign) unit direction vector of $\ell_{2}\left(\ell_{2}^{\prime}\right)$. Define $\Pi_{2}$ to be the plane passing through $p_{2}$ and spanned by the unit direction vectors $v_{2}$ and $v_{2}^{\prime}$. Define $\Pi_{3}$ to be the plane passing through $p_{3}$ and spanned by the unit direction vectors $v_{3}$ and $v_{3}^{\prime}$.

Figure 3 shows the choices of $\ell_{1}^{\prime}, \ell_{2}^{\prime}, \ell_{1}, \ell_{2}, \Pi_{0}, \Pi_{2}, \Pi_{3}, p_{2}, p_{3}$.

\subsection{Step 2. (Unit normals, $n_{1}, n_{2}, n_{3}$, and unit tangents $t_{1}, t_{2}, t_{3}$ )}

If necessary we change the sign of $v_{1}^{\prime}, v_{2}^{\prime}, v_{3}^{\prime}$ so that they point in the ventral direction. The 'normal' unit vector, $n_{j}$, associated to $p_{j}$ is chosen as $v_{j}^{\prime}$, $j=1,2,3$. The 'tangent' unit vector, $t_{j}$, associated to $p_{j}$ is chosen as the apically pointing normal vector of the plane $\Pi_{j}, j=1,2,3$.

This concludes the definition of the data $\left\{p_{j}, n_{j}, t_{j}, \Pi_{j}, j=1,2,3\right\}$. Figure 4 shows as an example the set of data for the first two adjacent lumbar vertebrae.

(The technique of using wedge properties of the vertebrae in precisely two directions to prescribe approximating planes for the top and bottom of a vertebra was recently used in a procedure described in Daghighi et al. (2018) in the context of obtaining artificial geometrical semi-global models.) 

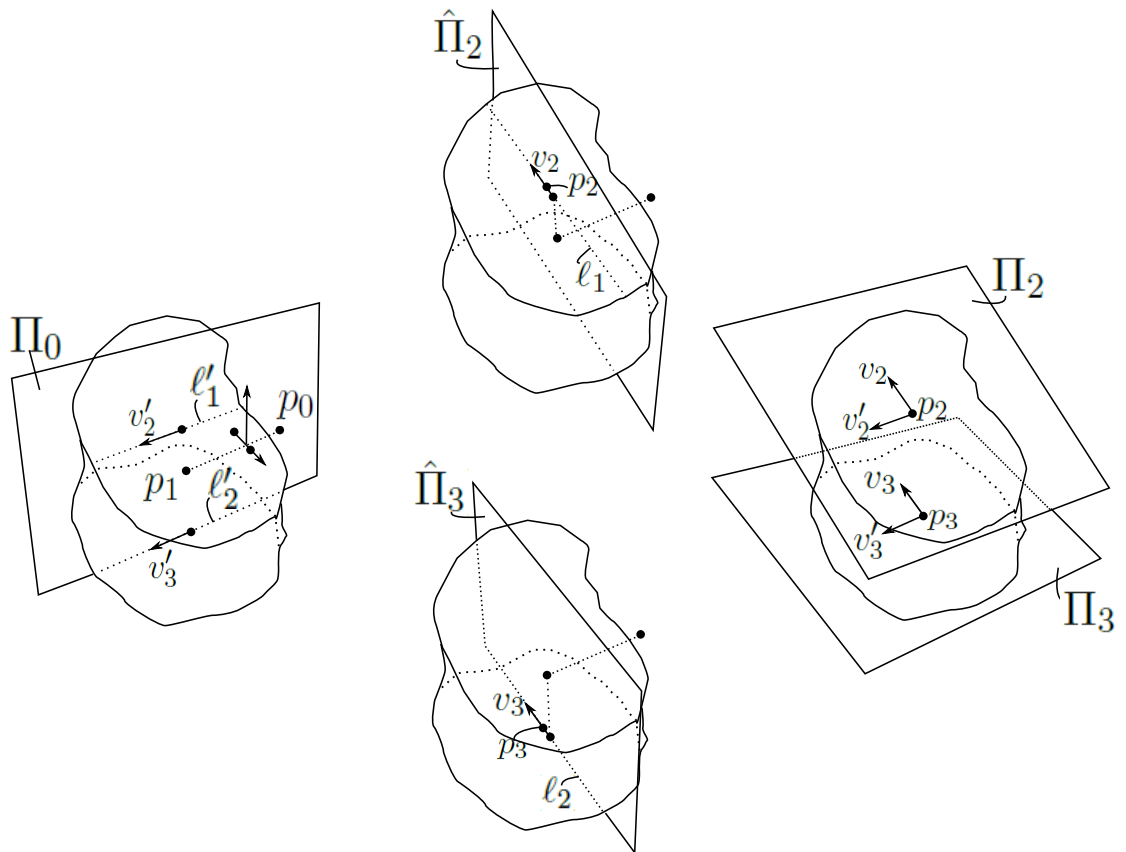

Figure 3. The choices of $\ell_{1}^{\prime}, \ell_{2}^{\prime}, \ell_{1}, \ell_{2}, \Pi_{0}, \Pi_{2}, \Pi_{3}, p_{2}, p_{3}$ obtained in Step 1 (Section 4.1). The notations are described in detail in the main text of Step 1

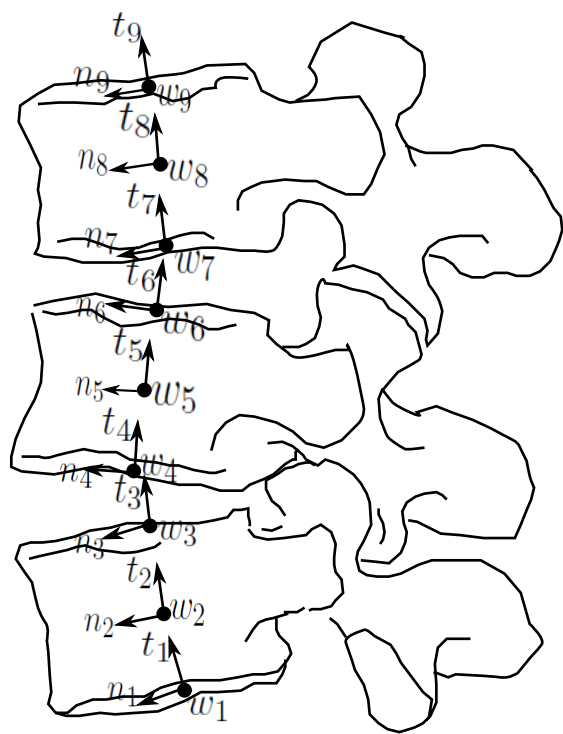

Figure 4. Set of data for the first three adjacent lumbar vertebrae. This is an illustration of the normals and tangents obtained in Step 2 (Section 4.2) 


\subsection{Step 3. (The estimates for curvature and torsion)}

Let us order the points from Step 1 as follows:

$\left(w_{1}, \ldots, w_{N}\right):=\left(p_{3}^{L_{5}}, p_{1}^{L_{5}}, p_{2}^{L_{5}}, p_{3}^{L_{4}}, \ldots, p_{3}^{T 1}, p_{1}^{T 1}, p_{2}^{T 1}\right), N=22 \cdot 3=66$, where for example $p_{3}^{L_{5}}$ denotes the point $p_{3}$ from Step 1 with respect to the vertebra $L 5$.

A regular $C^{\infty}$-smooth curve $\gamma: \mathbb{R} \rightarrow \mathbb{R}^{3}, \nu \mapsto \gamma(\nu)$, that passes through a set of points $w_{1}, \ldots, w_{N}$ for a positive integer $N$ can be written $\gamma(\nu)=$ $\left(\gamma_{1}, \gamma_{2}, \gamma_{3}\right)(\nu)$ for $C^{\infty}$-smooth components $\gamma_{j}, j=1,2,3$, such that for all $\nu$, $\frac{\partial \gamma}{\partial \nu} \neq 0$. In particular all of its three components are $C^{\infty}$-smooth functions of the variable $\nu \in \mathbb{R}$, and there are no singularities. Recall that having the tangent and normal at a given point means that we have the so-called osculating plane at that point. Recall that the curvature at a point $\nu_{0}$ of $\gamma$ is (up to sign) $\frac{1}{\left\|\frac{\partial \gamma\left(\nu_{0}\right)}{\partial \nu}\right\|^{3}}\left\|\frac{\partial \gamma\left(\nu_{0}\right)}{\partial \nu} \times \frac{\partial^{2} \gamma\left(\nu_{0}\right)}{\partial \nu^{2}}\right\|$ and the torsion at $\nu_{0}$ of $\gamma$ is $\left\|\frac{1}{\partial \nu} \times \frac{\partial^{2}}{\partial \nu^{2}}\right\| \operatorname{Det}\left(\frac{\partial \gamma}{\partial \nu}, \frac{\partial^{2} \gamma}{\partial \nu^{2}}, \frac{\partial^{3} \gamma}{\partial \nu^{3}}\right)\left(\nu_{0}\right)$ where $\operatorname{Det}(\cdot)$ denotes the determinant. Let us assume that $\gamma$ passes through the set of points $w_{1}, \ldots, w_{N}$. When $\gamma$ can be assumed to be parametrized by arc length the curvature at $\nu_{0}$ can (up to sign) be calculated as $\left\|\frac{\partial \hat{t}\left(\nu_{0}\right)}{\partial \nu}\right\|$ and the torsion at $\nu_{0}$ can (up to sign) be calculated as $\left\|\frac{\partial(\hat{t} \times \hat{n})\left(\nu_{0}\right)}{\partial \nu}\right\|$, where $\hat{n}(\nu)$ denotes the unit normal at $\nu$ and $\hat{t}(\nu)$ denotes the unit tangent at $\nu$. We keep the original point set $w_{1}, \ldots, w_{N}$ and assume arc length parametrization with parameter $\nu \in \mathbb{R}$. In particular there exist points $\nu_{1}, \ldots, \nu_{N}, \nu_{j}+1>\nu_{j}, j=1, \ldots, N-1$ such that $\gamma\left(\nu_{j}\right)=w_{j}$.

We use the following central difference scheme for (up to sign) the first order derivatives:

$$
\begin{aligned}
\kappa_{j} & :=\left\|\frac{t_{j+1}+t_{j-1}-2 t_{j}}{\nu_{j+1}-\nu_{j-1}}\right\|, \\
\tau_{j} & :=\left\|\frac{t_{j+1} \times n_{j+1}+t_{j-1} \times n_{j-1}-2 t_{j} \times n_{j}}{\nu_{j+1}-\nu_{j-1}}\right\| .
\end{aligned}
$$

It remains (under the assumption of arc length parametrization) to make an appropriate choice for the points $\nu_{j}, j=1, \ldots, N$. Since $\nu$ is supposed to be arc length, i.e. $\left\|\frac{\partial \gamma(\nu)}{\partial \nu}\right\| \equiv 1$, we can interpolate, using the tangents associated to the starting point set $w_{1}, \ldots, w_{N}$, new points iteratively and 


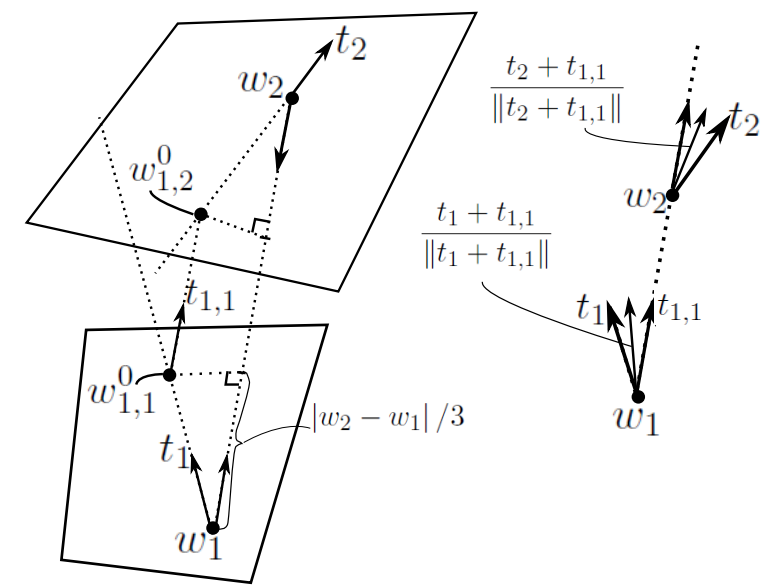

Figure 5. Interpolated points and tangents after two iterations starting from the first two points $w_{1}, w_{2}$ (with associated tangents $t_{1}, t_{2}$ ) in the process of approximating the arc length of $\gamma$ between these points. This is an illustration of the process described in detail in Step 3 (Section 4.3)

obtain an estimate of the arc length of $\gamma$ between each pair $w_{j+1}-w_{j}$, $j=1, \ldots, N-1$.

The interpolation will be done piecewise linearly, as follows: For two vectors $\alpha_{1}, \alpha_{2}$ in $\mathbb{R}^{3}$ recall that the orthogonal projection of $\alpha_{1}$ on $\alpha_{2}$ is $\alpha_{2} \frac{\alpha_{1} \bullet \alpha_{2}}{\alpha_{2} \bullet \alpha_{2}}$, where $\bullet$ denotes the Euclidean inner product on $\mathbb{R}^{3}$. Starting from the pair of points $\left(w_{j}, w_{j+1}\right)$, introduce the two interpolated points $w_{j, 1}^{0}:=$ $w_{j}+\frac{\left|\left(w_{j+1}-w_{j}\right) \bullet t_{j}\right|}{3} t_{j}, w_{j, 2}^{0}:=w_{j+1}+\frac{\left|\left(w_{j+1}-w_{j}\right) \bullet t_{j+1}\right|}{3}\left(-t_{j+1}\right)$.

Set $t_{j, 1}:=\frac{w_{j, 2}^{0}-w_{j, 1}^{0}}{\left\|w_{j, 2}^{0}-w_{j, 1}^{0}\right\|}$. Associate to $w_{j, 1}^{0}$ the tangent vector $\frac{1}{\left\|\frac{1}{2}\left(t_{j}+t_{j, 1}\right)\right\|}$ $\frac{1}{2}\left(t_{j}+t_{j, 1}\right)=\frac{t_{j}+t_{j, 1}}{\left\|t_{j}+t_{j, 1}\right\|}$ and associate to $w_{j, 2}^{0}$ the tangent vector $\frac{t_{j+1}+t_{j, 1}}{\left\|t_{j+1}+t_{j, 1}\right\|}$. This yields the augmented point set $w_{1}, w_{1,1}^{0}, w_{1,2}^{0}, w_{2}, \ldots, w_{N-1}, w_{N-1,1}^{0}$, $w_{N-1,2}^{0}, w_{N}$. The process can now be repeated using the pairs $\left(w_{1}, w_{1,1}^{0}\right)$, $\left(w_{1,1}^{0}, w_{1,2}^{0}\right), \ldots,\left(w_{N-1,1}^{0}, w_{N-1,2}^{0}\right),\left(w_{N-1,2}^{0}, w_{N}\right)$.

For example, in the second iteration, $w_{1}, w_{1,1}^{0}, w_{1,2}^{0}, w_{2}$ is replaced by $w_{1}, w_{1,1}^{1}, w_{1,2}^{1}, w_{1,1}^{0}, w_{1,3}^{1}, w_{1,4}^{1}, w_{2}$ and so on.

Figure 5 shows as an example the interpolated points and tangents after two iterations starting from the first two points $w_{1}, w_{2}$ (with associated tangents $\left.t_{1}, t_{2}\right)$ in the process of approximating the arc length of $\gamma$ between these points.

The process can easily be implemented in Matlab, and the user can 
choose the number of iterations. For example, after the first iteration the estimated arc length becomes $\mathbf{A}\left(w_{j+1}, w_{j}\right):=\left\|w_{j}-w_{j, 1}^{0}\right\|+\left\|w_{j, 1}^{0}-w_{j, 2}^{0}\right\|+$ $\left\|w_{j, 2}^{0}-w_{j+1}\right\|$. And for $m>m^{\prime}$

$$
\mathbf{A}\left(w_{m}, w_{m^{\prime}}\right)=\mathbf{A}\left(w_{m^{\prime}}, w_{m^{\prime}+1}\right)+\cdots+\mathbf{A}\left(w_{m-1}, w_{m}\right)
$$

We shall thus use the following estimates:

$$
\begin{aligned}
& \kappa_{j}:=\left\|\frac{t_{j+1}+t_{j-1}-2 t_{j}}{\mathbf{A}\left(w_{j+1}, w_{j-1}\right)}\right\| \\
& \tau_{j}:=\left\|\frac{t_{j+1} \times n_{j+1}+t_{j-1} \times n_{j-1}-2 t_{j} \times n_{j}}{\mathbf{A}\left(w_{j+1}, w_{j-1}\right)}\right\|
\end{aligned}
$$

Finally we replace $\kappa_{j}$ with $\operatorname{sign}\left(\kappa_{j}\right)\left|\kappa_{j}-\hat{\kappa}_{j}\right|$ in order to obtain what we call the local effective curvature at $p_{j}$, and we do this for all the points (ordered caudally to apically) $v_{k}, k=2, \ldots, N-1$.

\subsection{Step 4}

Definition 4.2 (Local effective deformation). The local effective deformation associated to the point $p_{j}$ is defined as $\left|\left(\kappa_{j}, \tau_{j}\right)\right|, j=2, \ldots, N-1$. When the correction using the $\hat{\kappa}_{j}$ is not used, we shall call $\kappa_{j}$ the noncorrected local curvature at $p_{j}$, and $\operatorname{sign}\left(\kappa_{j}\right)\left|\kappa_{j}-\hat{\kappa}_{j}\right|$ is called the corrected local effective curvature at $p_{j}$.

In order to find an estimate for the curvature or torsion of a segment of the vertebral body (e.g. between two end-plates that are used to calculate the Cobb angle) we sum over all local curvatures at points within that segment.

Definition 4.3 (Segmental effective deformation). The segmental effective deformation for the segment of the vertebral column starting at the lower vertebra $X_{1}$ and ending at the vertebra $X_{M}$, for a positive integer $M$, where $X_{1}, \ldots, X_{M}$ is a subset (with the inherited order) among the ordered set $L_{5}, L_{4}, \ldots, T_{2}, T_{1}$, is defined as the sum $\sum_{k, j}\left|\left(\kappa_{X_{k, j}}, \tau_{X_{k, j}}\right)\right|$, where $\kappa_{X_{k, j}}$ denotes $\kappa_{j}$ calculated with respect to the point $p_{j}$ associated to the vertebra $X_{k}$. When the correction using the $\hat{\kappa}_{j}$ is not used, we shall use the term non-corrected segmental effective curvature of the segment $X_{1}$ to $X_{M}$. 
Case exception clause for abnormal anatomy: In the case of abnormal anatomy (e.g. congenital or previous operation which has disrupted the anatomical landmarks rendering them incompatible with one of the steps above) we include a case exception, where any such vertebra under consideration is modelled by the procedure described in detail in our previous work, Daghighi et al. (2018), Section 3.3, Figures 5 and 6 (these are called artificial geometric models of vertebrae). In particular the artificial geometric modelling renders an ellipse in a two-dimensional plane for both the top and bottom of the modelled vertebra, hence there is a unique well-defined unit normal vector assigned to the top and bottom respectively; these are taken as $t_{1}, t_{2}, t_{3}$ (cf. Step 2), and furthermore the simple geometric shape of these models makes it natural to choose the centres of the aforementioned ellipses together with the centre of mass of the modelled vertebra to play the role of the three points $\left(p_{1}, p_{2}, p_{3}\right.$, cf. Step 1) which the method of the present article assigns to vertebrae with normal anatomy. To obtain replacements of vectors $n_{1}, n_{2}, n_{3}$ we only need to specify a direction corresponding to an approximation of axial rotation, and for this we use the point in the vertebral groove (or an approximation of it) with minimal distance to $p_{1}$ (such a point will play the role of $p_{0}$ in the above method). This gives, together with any two of $p_{1}, p_{2}, p_{3}$, a plane which plays the role of $\Pi_{0}$, whence a natural choice of $n_{1}$ - and similarly for $n_{2}$ and $n_{3}$. Obviously, the case exception procedure can be repeated for each vertebra which has an anatomical anomaly that disrupts the procedure of the present article (e.g. if a previous scoliosis operation with pedicle screws has been performed, this usually involves several vertebrae).

\section{Cobb angle equivalence classes}

In order to illustrate why we find Cobb angle insufficient as a measure of deformation, we introduce an equivalence relation on the set of 3D models of vertebral columns.

Definition 5.1. To each theoretical anatomically possible vertebral column segment L5-T1, we associate its isolated skeletal 3D segmentation (i.e. surrounding soft tissue, vertebral discs and ribs being removed), and to each such object we associate the chain of isolated vertebral bodies (i.e. with lamina, processus spinosus, processus transversus, etc. removed) and denote the set of objects (segmentations) so obtained as $\mathcal{S}$. Assume that there exists a rigorously reproducible process for taking a frontal X-ray, in the 
sense that the patient can be assumed always to have precisely one posture during the scan representative of their habitual state. Order the set of vertebrae $L_{5}, L_{4}, \ldots, T_{2}, T_{1}$ and rename them according to $X_{1}, \ldots, X_{N}$, where in normal anatomy $N=17$. Let $X \in \mathcal{S}$ be such that the underlying vertebral column for $X$, in a rigorously (in the above sense) obtained clinical $\mathrm{X}$-ray, has apex vertebra $X_{n}$ and Cobb angle $\theta$ calculated with respect to the end-plates of the end vertebrae $X_{n+j}, X_{n-k}$, for positive integers $j, k$ such that $1 \geqslant n-k \leqslant N, 1 \geqslant n+j \leqslant N$. An element $Y \in \mathcal{S}$ is called Cobb angle equivalent to $X$, denoted $X \sim Y$, if $Y$ in a rigorously obtained clinical $\mathrm{X}$-ray has the same apex vertebra $X_{n}$, the same orientation (right or left convex), and Cobb angle $\theta$ calculated with respect to the same end-plates $\left(X_{n+j}, X_{n-k}\right)$. Clearly (i) $X \sim X$, (ii) $X \sim Y \Rightarrow Y \sim X$, (iii) if $Z \in \mathcal{S}$ then $X \sim Y \vee Y \sim Z \Rightarrow X \sim Z$. Thus $\sim$ is an equivalence relation, and we denote by $[X]$ the equivalence class of all members of $\mathcal{S}$ that are equivalent (with respect to $\sim$ ) to $X$.

The quotient space $\mathcal{S} / \sim$ is what one is considering in clinical practice when using merely the Cobb angle. But it is clear that each $[X]$ is a large set containing members corresponding to vertebral columns with greatly varying 3D deformations. We shall for our purposes pick a representative (called the canonical representative) whose segment underlying the calculation for the Cobb angle of the largest appearing scoliosis curve is particularly simple. We do this with less attention to how the remaining parts of the vertebral column become represented as long as the choice is unique, since we shall only use the first mentioned segment for our comparisons in future work.

Definition 5.2 (Cobb associated deformation). In order to be able to compare our deformation measure to Cobb angle measurements in future work, we shall to each $X \in \mathcal{S}$ associate the unique member $\hat{X} \in[X]$ ( $\hat{X}_{\text {corr }} \in[X]$ respectively), called the canonical representative, such that: (i) $\hat{X}$ has zero kypholordosis (or 'healthy' values $\hat{\kappa}$ respectively) in the segment $X_{n-k}, X_{n-k+1}, \ldots, X_{n+j}$, used for the Cobb angle. (ii) Each vertebra $X_{n-k}, X_{n-k+1}, \ldots, X_{n+j}$, has zero axial rotation. (Note that (i) and (ii) together imply that the normals and tangents from the procedure for measuring segmental effective deformation can be obtained without the use of the anatomical landmarks previously required, hence it is sufficient to consider only the vertebral bodies in the representation.) (iii) The points of the procedure for measuring segmental effective deformation (as presented in this paper) for the segment $X_{n-k}, X_{n-k+1}, \ldots, X_{n+j}$, lie on a circular 
$\operatorname{arc} \mathcal{C}$, and the middle point of the segment coincides with the middle point $p_{1}$ associated to the apex vertebra according to the procedure described in this text. The canonical representative $\hat{X}$ is stipulated to have length $|\mathcal{C}|$ given by $m \cdot 4 \mathrm{~cm}$, where $m$ is the number of vertebrae in the main scoliosis curve. (iv) The segments $X_{1}, \ldots, X_{n-k}$, and $X_{n+j}, \ldots, X_{N}$, have the points in the procedure for measuring segmental effective deformation, following circular arcs of half and opposite curvature, and in the same plane, as the arc chosen for the segment $X_{n-k}, X_{n-k+1}, \ldots, X_{n+j}$. (v) The vertebrae for the segment $\mathcal{C}$ are obtained by starting from a thickening of the circular arc, to a cylindrical solid with diameter $10 \mathrm{~cm}$, and removing, at equally spaced angles, a segment corresponding to 4 degrees for each vertebral disc (see Figure 6). (One may choose the size of the thickening arbitrarily, and each fixed choice will provide an applicable equivalence relation. It is clear that there is no need to vary the diameter along the vertebral column, as the equivalence relation is based upon the curvature. We have chosen $10 \mathrm{~cm}$ for the diameter, as this seems to be an appropriate average measure.) The angle, $\varphi$, corresponding to each vertebra is determined by the subdivision of the middle circular arc based upon the number of vertebrae used to calculate the Cobb angle. (vi) The vertebrae for the remaining two segments are obtained by starting from a thickening of the circular arcs to a cylindrical solid with diameter $10 \mathrm{~cm}$, and removing, at equally spaced angles of $\frac{\varphi}{2}$, a segment corresponding to 2 degrees for each vertebral disc. (see Figure 6). Note that for a given Cobb angle $\theta$ and a given sector length $|\mathcal{C}| \mathrm{cm}$, we have a corresponding circle with a radius which we denote $\rho \mathrm{cm}$ (thus depending upon the number of vertebrae $m$ in the main scoliosis curve and on the Cobb angle), such that $|\mathcal{C}|=\rho \cdot(\theta \cdot 2 \pi / 360)$.

This choice of $\hat{X} \in[X]$ ( $\hat{X}_{\text {corr }} \in[X]$ respectively) is by construction unique with respect to $\mathcal{S}$ (in the set of bone segmented vertebral columns it is only unique up to different shapes of all parts of the vertebrae except the vertebral body).

The segmental effective deformation of $\hat{X}$ ( $\hat{X}_{\text {corr respectively) for the seg- }}$ ment $X_{n-k}, X_{n-k+1}, \ldots, X_{n+j}$, is called the Cobb associated deformation (corrected Cobb associated deformation respectively) of the vertebral column representative $X$.

Clearly, we can use the corrected Cobb associated deformation when performing a study of comparison to the segmental effective deformation of a given segment that is used for Cobb angle measurement. 


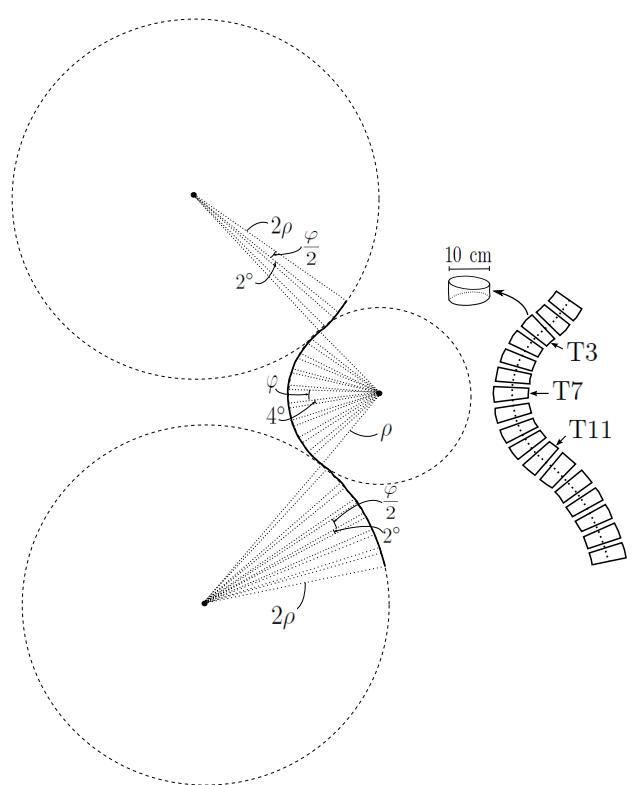

Figure 6. The representative of $X$ used for Cobb associated deformation in the sense of Definition 5.2, for the case where $X$ has apex at $T 7$ with associated Cobb angle using end vertebrae $T 3$ and $T 11$ respectively. In this case the number $m$ in Definition 5.2 (i.e. the number of vertebrae involved in the definition of the main scoliosis curve) is 9 , so $\rho=(9 \cdot 4 \mathrm{~cm}) /(\theta \cdot 2 \pi / 360$ radians $)$

Remark 5.3. Note that the chosen representative $\hat{X}$ is adapted to the major scoliosis curve, i.e. (since it has piecewise constant curvature) the segment underlying the calculation for the Cobb angle of the largest appearing scoliosis curve. We do this with less attention to how the remaining parts of the vertebral column become represented, since we shall only use the first mentioned segment for our comparisons in future work. What is important is that the choice of representative is unique and particularly simple in the region of interest.

Figure 6 shows the representative of $X$ used for Cobb associated deformation in the sense of Definition 5.2, for the case where $X$ has apex at $T 7$ with associated Cobb angle using end vertebrae $T 3$ and $T 11$ respectively; notice how only the sagittal wedge properties of the vertebrae are relevant. As this text is intended to be a technical method article, no patient consents for publication of CT extracted data are available, although it should be perfectly clear for any reader who has a working experience of the MPR application in the PACS system that by adjusting the planes in three dimen- 
sions, all the required points for each of the steps in the algorithm are easily obtainable, and the algorithm is tailored for these to be essentially unique (up to measurement errors and certain natural user variation) for a given CT scan. Our goal is to present explicit calculations pre-and post-operatively after presenting an upcoming paper regarding appropriate normal values of lordokyphosis. The latter will provide the obligatory prerequisites needed to obtain the corrected effective curvature.

For the reader's convenience, however, we may use the definition of Cobb angle equivalence classes to present an example which does not require any patient CT scan, because we shall base our values on an elementary geometric model, in fact one obtained from a canonical representative of a Cobb angle equivalence class.

Example 5.4. Recall that for a given thoracic Cobb angle with associated orientation (right or left convex) and fixed apex and end-plates for the curve associated to the Cobb angle, we have a canonical representative of the Cobb angle equivalence class. Consider the vertebral column given by the canonical representative of the Cobb angle equivalence class of vertebral columns with a right-convex Cobb angle of 10 degrees, with apex at $\mathrm{T} 7$ and end-plates T4 and T10. In this case the number $m$ in Definition 5.2 (i.e. the number of vertebrae involved in the definition of the main scoliosis curve) is 7 , so we have an induced circle (see Figure 6 ) with radius $\rho=(7 \cdot 4 \mathrm{~cm}) /(\theta \cdot 2 \pi / 360$ radians $)$, where $\theta=10$ degrees. Note that the canonical representative, by (i) of Definition 5.2, has no lordokyphosis. We shall see that, by Definition 5.2, a simple modification of the canonical representative, we obtain a vertebral column for which the full $\mathrm{CT}$ algorithm is greatly reduced. Fix the origin $(0,0,0)$ in $x y z$-space to lie at the centre of the aforementioned circle with radius $\rho$ (note that this circle lies in the $x z$-plane). Technically, to be able to apply the algorithm we need some anatomical landmarks. Note that the set of points of a given vertebra of the canonical representative (in the given coordinate system) that intersect the plane $\{y=-5 \mathrm{~cm}\}$ forms a curve segment, $\alpha$, in that plane $\{y=-5 \mathrm{~cm}\}$, which is a translate of a segment of the initial circular arc in Definition 5.2 of the canonical representative. Thus we adjoin to the vertebral body a curved cylindrical shell obtained from a thickening of $\alpha$, and this will simulate a lamina enclosing the vertebral canal. This will induce, in Step 1 (Section 4.1), symmetrically placed $\eta_{1}, \ldots, \eta_{4}$, thus giving symmetrically placed $\eta_{5}, \eta_{6}$ such that the procedure of Step 1 (Section 4.1) will give $p_{1}$ lying in the middle of the two-dimensional slice through the centre of mass 
of the simple geometric shape of the vertebral body. By (ii) of Definition 5.2 the axial rotation is zero, thus the contribution of $\tau$ is zero; in particular we may assume that the three-dimensional coordinates $x y z$ are chosen such that for any given vertebra under consideration, the associated vectors $t_{1}, t_{2}, t_{3}$ all have zero $y$-component, so that $n_{1}, n_{2}, n_{3}$ each coincide with the vector $(0,1,0)$. In essence we need only consider the $x z$-plane. Note that the $t_{i}$ become normalizations of the cross products $\left(p_{i}-(0,0,0)\right) \times(0,1,0)$, $i=1,2,3$. We have, by the well-defined elementary geometric shape of the vertebrae in the canonical representative and their symmetry, that the coordinates required for the calculation of the effective local curvature are, for e.g. the apex: $p_{1}=\rho(\cos (\pi), 0, \sin (\pi)), p_{2}=\rho\left(\cos \left(\pi-\theta_{0}\right), 0, \sin \left(\pi-\theta_{0}\right)\right)$, $p_{3}=\rho\left(\cos \left(\theta_{0}\right), 0, \sin \left(\theta_{0}\right)\right)$, where $\theta_{0}:=\frac{4}{2} \cdot \frac{\pi}{360}$ radians (cf. Figure 6$)$ and $\rho$. We now have all the required inputs $p_{1}, p_{2}, p_{3}, t_{1}, t_{2}, t_{3}, n_{1}, n_{2}, n_{3}$, which can be plugged into the definition of the non-corrected local effective curvature $\kappa_{7}$ at $p_{1}$ (corresponding to the middle one of the three points associated to the apex (T7)).

\section{Discussion}

We believe that, although there currently exist measures of scoliosis which are very good in practice, they do not reflect the three-dimensional deformity near the apex in an optimal fashion (from a theoretical point of view, despite e.g. sagittal modifiers). One motivation to accept our proposed methodology and results is the following. Technical applications are usually best manifested in terms of a rigorous and formal mathematical model. This involves thorough definitions (with minimal ambiguity). From a theoretical point of view it makes sense to include the notion of curvature and torsion in describing the three-dimensional deformation of a vertebral column. Focus has been placed on developing steps for the construction of the points involved which are reproducible and well-defined (in particular, one goal has been that they yield unique points of reference which are then used in the calculations of the proposed deformation parameter). Also the method is developed in such a way that all steps can be performed using existing modern roentgenological software. A clear limitation of the resultant definition is that it is based upon CT scans, and also that it requires the use of the application MPR (multi-phase reconstruction). This means that in order to obtain the parameter in practice the patient must be exposed to radiation, and the MPR application is a rather recent addition to the 
user interface of IDS-7 from Sectra, and thus is perhaps not internationally available clinically. A second limitation is of course that (as we have pointed out) the general deformation measure obtained should from a logical point of view be corrected for the fact that there exists a physiological lordokyphosis. We have presented how this can be done, but we currently do not have consensus international reference values for (age and weight normalized) 'normal values'; see the section on future work.

\section{Future work}

In this work we have chosen not to include any patient data or images, thus making it a theoretically based technical report instead of a clinical study. All required data points can, however, be obtained relatively easily and quickly using the MPR application in IDS-7, PACS allowing the practical implementation of the described method. In future work we propose to use $\mathrm{CT}$ for a sufficient number of patients with healthy vertebral columns in order to derive estimates for the normal local kypholordosis (in the absence of vertebral rotation) at each vertebral level (this will provide the values $\hat{\kappa}$ required to calculate the corrected version of the deformation parameter). Then we propose to perform a study on a sufficient number of scoliosis patients before operation, with varying Cobb angle in a relatively large range. For these patients we shall then compare our deviation measure on the segment defined by the end vertebrae (end-plates) used to define the Cobb angle, with the curvature derived from the Cobb angle (in terms of Cobb associated deformation), and further investigate whether or not the maximum deviation for our adjusted parameter occurs at the apex.

\section{Acknowledgements}

The research was partially supported by an ALF grant (Event No:LIO608221). The authors thank the referees and the editor for helpful comments.

\section{REFERENCES}

Daghighi A., Tropp H., Dahlsröm N., Klarbring A.: A F.E.M. stress investigation of scoliosis apex. The Open Biomedical Engineering Journal 12: 51-71.

D'Amico M., Merolli A., Santambrogio G.C. (eds) (1995): Three Dimensional Analysis of Spinal Deformities. IOS Press.

Dansereau J., Stokes I. (1988): Measurements of the three-dimensional shape of the rib cage. Journal of Biomechanics 2(11): 893-901. 
Donzelli S., Poma S., Balzarini L., Borboni A., Stefano Respizzi S., Villafane J.H., Zaina F., Negrini S. (2015): State of the art of current 3-D scoliosis classifications: a systematic review from a clinical perspective. J. Neuroeng. Rehabil. 12: 91.

Drerup B., Hierholzer E. (1992): Evaluation of frontal radiographs of scoliotic spines-Part I measurement of position and orientation of vertebrae and assessment of clinical shape parameters. Journal of Biomechanics. Technical note 25(11): 1357-1362.

Ho E., Upadhyay S.S., Chan F.L., Hsu L., Leong J. (1993), New Methods of Measuring Vertebral Rotation From Computed Tomographic Scans: An Intraobserver and Interobserver Study on Girls with Scoliosis. Spine 18: 1173-1177.

Kawakami N., Tsuji T., Imagama S., Lenke L.G., Puno R.M., Kuklo T.R. (2009): Classification of Congenital Scoliosis and Kyphosis: A New Approach to the Three-Dimensional Classification for Progressive Vertebral Anomalies Requiring Operative Treatment. Spine 34(17): 1756-1765.

Lam G.C. (2008): Vertebral rotation measurement: a summary and comparison of common radiographic and CT methods. Scoliosis 3(16): 1-10.

Lenke L.G., Betz R.R., Harms J., Bridwell K.H., Clements DH., Lowe T.G., Blanke K.(2001): Adolescent idiopathic scoliosis: a new classification to determine extent of spinal arthrodesis. Journal of Bone and Joint Surgery, American Volume. 83-A(8): 1169-1181.

Ovadia D. (2013): Classification of adolescent idiopathic scoliosis. Journal of Children's Orthopaedics 7(1): 25-28.

Sangole A., Aubin C.-E., Labelle H., Stokes I.A., Lenke L.G., Jackson R., Newton P. (2009): Three-dimensional classification of thoracic scoliotic curves. Spine 34(1): 91-99.

Somoskeöy S., Tunyogi-Csapó M., Bogyó C., Illés T. (2012): Clinical validation of coronal and sagittal spinal curve measurements based on three-dimensional vertebra vector parameters. The Spine Journal 12(10): 960-968.

Stokes I. (1994): Scoliosis research society working group on 3D terminology of spinal deformity: Three-dimensional terminology of spine deformity. Spine 19: $236-248$.

Stokes I., Sangole A.P., Aubin C.E. (2009): Classification of scoliosis deformity three-dimensional spinal shape by cluster analysis. Spine 34(6): 584-590.

Vrtovec T., Pernus F., Likar B. (2009): A review of methods for quantitative evaluation of spinal curvature. European Spine Journal 18(5): 1-15. 